This item was submitted to Loughborough's Research Repository by the author.

Items in Figshare are protected by copyright, with all rights reserved, unless otherwise indicated.

\title{
Independent and interactive effects of thermal stress and mental fatigue on manual dexterity
}

\section{PLEASE CITE THE PUBLISHED VERSION}

https://doi.org/10.1152/ajpregu.00226.2020

\section{PUBLISHER}

American Physiological Society

VERSION

AM (Accepted Manuscript)

\section{PUBLISHER STATEMENT}

This paper was accepted for publication in the journal American Journal of Physiology-Regulatory, Integrative and Comparative Physiology and the definitive published version is available at https://doi.org/10.1152/ajpregu.00226.2020

\section{LICENCE}

CC BY-NC-ND 4.0

\section{REPOSITORY RECORD}

Valenza, Alessandro, Harry Charlier, Antonino Bianco, and Davide Filingeri. 2020. "Independent and Interactive Effects of Thermal Stress and Mental Fatigue on Manual Dexterity”. Loughborough University. https://hdl.handle.net/2134/13095872.v1. 
9 Type of submission: Research article

10 Running title: Interaction of thermal stress and mental fatigue on manual performance

11 Key words: Mental Fatigue, Hot Temperature, Cold Temperature, Hand, Physical Functional

12 Performance

13 Word count: 5793

14 Number of references: 44

15

16 Corresponding author:

17 Davide Filingeri $\mathrm{PhD}$

18 THERMOSENSELAB

19 Environmental Ergonomics Research Centre, Loughborough Design School

20 Loughborough University

21 Loughborough LE11 3TU UK

22 Phone: +4401509228169

23 E-mail: d.filingeri3@lboro.ac.uk manual dexterity University, Loughborough, UK

\author{
Palermo, Palermo, Italy
}




\section{Abstract}

28 Many occupations and sports require high levels of manual dexterity under thermal stress and mental fatigue. Yet, multi-stressor studies remain scarce. We quantified the interactive effects

30 of thermal stress and mental fatigue on manual dexterity. Seven males (21.1 $\pm 1.3 \mathrm{y})$ underwent 316 separate 60 -min trials characterised by a combination of 3 air temperatures (HOT: $37^{\circ} \mathrm{C}$; 32 NEUTRAL: $21^{\circ} \mathrm{C}$; COLD: $7^{\circ} \mathrm{C}$ ) and 2 mental fatigue states (MF: mental fatigue induced by a 33 35-min cognitive battery; No-MF: no mental fatigue). Participants performed complex 34 (O'Connor test) and simple (Hand-Tool test) manual tasks pre- and post-trials to determine 35 stressors-induced performance changes. We monitored participants' rectal temperature and 36 hand skin temperature ( $\mathrm{T}_{\text {hand }}$ ) continuously and assessed reaction time (Hand-Click test) and 37 subjective mental fatigue ( 5 -point scale). Thermal stress $(\mathrm{p}<0.0001)$, but not mental fatigue $38(\mathrm{p}=0.290)$, modulated $\mathrm{T}_{\text {hand }}\left(\mathrm{HEAT}:+3.3^{\circ} \mathrm{C}[95 \% \mathrm{CI}+0.2,+6.5]\right.$; COLD: $-7.5^{\circ} \mathrm{C}[-10.7$, 39 4.4]). Mental fatigue $(\mathrm{p}=0.021)$, but not thermal stress $(\mathrm{p}=0.646)$, slowed reaction time $40 \quad(\sim 10 \%)$ and increased subjective fatigue. Thermal stress and mental fatigue had an interactive 41 effect on the complex manual task ( $\mathrm{p}=0.040)$, with COLD-No-MF decreasing performance 42 by $-22 \%[-39,-5]$, while NEUTRAL-MF, COLD-MF, and HEAT-MF by -36\% [-53, -19], $4334 \%[-52,-17]$, and $-36 \%[-53,-19]$, respectively. Only mental fatigue decreased 44 performance in the simple manual task $(-30 \%[-43,-16]$ across all thermal conditions; $45 \mathrm{p}=0.002$ ). Cold stress-induced impairments in complex manipulation increase with mental 46 fatigue; yet combined stressors' effects are no greater than that of mental fatigue alone, which 47 also impairs simple manipulation. Mental fatigue poses a greater challenge to manual 48 dexterity than thermal stress. 


\section{Introduction}

51 Many occupations such as those in the military and healthcare sectors, as well as competitive 52 sports, require high levels of manual dexterity to ensure optimal performance. However, 53 these occupations and sports are often performed under conditions of thermal stress and mental fatigue, which pose a challenge for manual performance. A military doctor treating a gunshot wound during a cold-weather operation, an ICU nurse intubating a patient after a long shift and while wearing personal protective equipment, and a shooting athlete reloading a rifle during a hot summer Olympics' final, are all examples of real-life scenarios where optimal manual performance is essential, yet it may be impaired by the combined presence of thermal and cognitive stressors.

Manual dexterity is described as the ability to make coordinated hand and finger movements aimed at manipulating objects (21). This motor ability relies on neurophysiological (e.g. reflex motor control of grip force) as well as neurocognitive mechanisms (e.g. visuo-motor coordination, information processing and reaction time), and ranges from simpler to more complex actions (e.g. from simply unscrewing a bolt using the thumb and index finger to 65 pinch-gripping a needle and placing it in a small hole) (21).

66 It is well established that thermal stress, and particularly cold stress, has a detrimental effect 67 on manual dexterity (9). For example, manual dexterity worsens when local hand and finger temperatures decrease to $\sim 20^{\circ} \mathrm{C}$ as a result of cold air exposure (20), with this effect becoming more severe at hand skin temperatures of $15^{\circ} \mathrm{C}$ and below (9). Furthermore, localised heating of the hand increases tapping speed; yet it also delays simple reaction time (25). Local changes in hand and finger temperatures play a greater role in worsening manual performance than changes in core temperature, with manipulative performance being hardly influenced by decreases in core temperatures when the hand is kept warm $(8 ; 12)$. Changes in manual performance due to hand and fingers cooling are due to loss of cutaneous sensitivity (35), secondary to conduction slowing in cold cutaneous nerve afferents $(10 ; 41)$. Conduction velocity in cutaneous nerve fibers decreases linearly when skin temperature drops from 36 to $23^{\circ} \mathrm{C}$ (10); a more pronounced slowing occurs at temperatures below $20^{\circ} \mathrm{C}$ (41), with conduction blocks developing at skin temperatures $\sim 8^{\circ} \mathrm{C}$ (35). Furthermore, cooling (but not heating) of synovial fluid, reduces joint mobility, which can in turn contribute to thermalstress induced decrements in manual performance (22).

81 Beside thermal stress, cognitive stressors such as mental fatigue (i.e. a psychobiological state

82 induced by prolonged periods of demanding cognitive activity) $(11 ; 23)$ can also be 
83 detrimental to manual performance $(3 ; 13)$ by increasing perceived exertion (32) and 84 hindering cognitive and perceptual skills (14), including reaction time (25).

85 While the independent effects of thermal (i.e. heat and cold stress) and cognitive stressors 86 (i.e. mental fatigue) on manual performance have been studied in isolation, there is a paucity 87 of data on their combined effects on the dexterous execution of both simple and more 88 complex manual tasks. This opens to the question of which stressor, i.e. thermal stress or 89 mental fatigue, poses a greater challenge to manual dexterity, and how their effects combine 90 under a multi-stressor scenario. There is some evidence that heat stress and mental fatigue 91 can interact synergistically in reducing cycling endurance capacity (36); yet, multi-stressors 92 studies remain scarce, particularly in the context of manual performance. Quantifying the 93 independent and interactive effects of multi-stressor scenarios on performance is 94 fundamentally important to better understand human integrative physiological responses (27). 95 Furthermore, this basic knowledge can support the development of applied interventions 96 aimed at mitigating the impact of the more detrimental stressor, to preserve performance 97 more effectively.

98 The interactions between stressors depend on the mechanisms by which each stressor exerts 99 its effects $(5 ; 28)$. Stressors with largely independent mechanisms of action often induce 100 additive effects, while stressors that are mechanistically similar may induce synergistic or 101 antagonist effects (27). Both thermal stress and mental fatigue induce deterioration of 102 reaction time and hand motor coordination by either slowing peripheral conduction velocity 103 or engaging central cognitive resources. Accordingly, it would be reasonable to hypothesize 104 that those stressors may induce a synergistic deterioration of manual performance when 105 combined.

106 The aim of this study was to quantify the independent and interactive effects of thermal stress 107 and mental fatigue on manual performance. Our objectives were to assess manual dexterity 108 during simple and complex tasks following 60-min exposures to six combinations of three 109 environmental temperatures (i.e. 7, 21 and $37^{\circ} \mathrm{C}$ ) and two mental fatigue states (i.e. with and 110 without mental fatigue) in healthy young adults. We hypothesized that thermal stress and 111 mental fatigue would interact synergistically in impairing manual dexterity, i.e. their 112 combined impact would be greater than the sum of their individual impacts.

113

\section{Methods}

\section{Ethical approval}


116 The testing procedure and the conditions were explained to each participant and they all gave

117 written informed consent for participation. The study was approved by the Loughborough

118 University Ethics Sub-Committee for Human Participants, and testing procedures were in

119 accordance with the tenets of the Declaration of Helsinki. All testing took place in

120 Loughborough (UK) between June and September 2017.

121

\section{Participants}

123 Given the experimental demands of the present study (i.e. each participant would partake in 124 six separate 60-min trials in hot, cold, and mentally fatigued conditions), and in line with 125 similar research (see e.g. 28; 36), we used a convenience sampling approach to recruit eight 126 non-smoking, recreationally active males (i.e. more than/or 3 exercise sessions per week) 127 with no neurological, cardiovascular nor sensory-related disorders (e.g. numbness over the 128 hands), and with normal or corrected-to-normal vision, were recruited from the student 129 population of Loughborough University to take part in this study.

130 Due to technical reasons, only 7 participants (age $20.6 \pm 1.3 y$; body fat $12.7 \pm 2.1 \%$; height $131 \quad 180.3 \pm 4.1 \mathrm{~cm})$ completed all experimental trials. In the $24 \mathrm{~h}$ prior to each trial, participants 132 were instructed to refrain from: 1) performing mental and physical training; 2) consuming 133 caffeine, alcohol or any other stimulant. All participants performed their trials at the same 134 time of the day. As we appreciated the limitations of a convenience sampling approach, we 135 performed a sensitivity analysis to determine the required effect size and $\mathrm{F}$ critical for a 136 statistically significant interaction between thermal stress and mental fatigue for the complex 137 manual task (i.e. ANOVA repeated measure, within-between interaction), given our sample 138 size of 7 , an $\alpha$ of 0.05 , a $\beta$ of 0.95 , a number of groups of 6 , and a number of measurements 139 of 12. We determined those values to be 1.09 (effect size f) and 2.49 (F critical) and we 140 therefore used these parameters as a threshold for interpreting our findings. The power 141 analysis was performed in GPower 3.1.9.2.

143 Experimental design

144 We used a single-blind, repeated measure design, where participants were made aware of the 145 purpose of the study, but not of its hypotheses nor the characteristics of each experimental 146 trial (e.g. ambient temperature and induction of mental fatigue), to limit any expectation bias.

147 All participants took part in six different 60-min trials on separate days, which were 148 characterised by a different combination of three ambient temperatures (HEAT: $37.0 \pm 0.4^{\circ} \mathrm{C}$; 149 NEUTRAL: $21.0 \pm 0.4^{\circ} \mathrm{C}$; COLD: $7.0 \pm 0.4^{\circ} \mathrm{C}$; relative humidity maintained at $40.0 \pm 1.5 \% \mathrm{RH}$ 
150 for all temperatures) and two mental fatigue states (MF: mental fatigue; No MF: no mental 151 fatigue) (Fig. 1). At the beginning (i.e. baseline) and at the end (i.e. post-test) of each trial 152 participants performed a complex manual task, i.e. O'Connor finger dexterity test (O'Connor 153 dexterity test, 2017), and a simpler manual task, i.e. modified Hand-Tool Dexterity test 154 (Hand-Tool Dexterity test, 2017). We introduced both simpler and more complex manual 155 dexterity tasks to provide a more comprehensive assessment of the potential interactive 156 effects of our chosen stressors, as well as for their relevance to occupational settings (e.g. a 157 soldier controlling a drone or reloading a gun). The mode of execution of both tests 158 administered falls within a general assessment of manual dexterity, which depending on the 159 task may require more complex vs. simpler motor actions.

160 During all trials, participants rested in a seated position in a climatic chamber, while wearing 161 a standardised clothing ensemble consisting of T-shirt, running shorts, socks and running 162 shoes. We monitored participants' whole-body thermal state [i.e. rectal temperature $\left(\mathrm{T}_{\text {rec }}\right)$, 163 hand skin temperature $\left(\mathrm{T}_{\text {hand }}\right)$, and upper body mean skin temperature $\left.\left(\mathrm{T}_{\text {upper body }}\right)\right]$ and 164 cognitive state (i.e. reaction time and subjective mental fatigue), in order to quantify the 165 impact of each stressor on participants' physiological and cognitive responses. All trials were 166 performed in a counter-balance order to avoid any order effect.

167 By comparing participants' change in manual performance between pre- and post-test in each 168 trial with the pre-to-post change in performance in the NEUTRAL-No MF trial (i.e. namely 169 the control condition), this design allowed for the quantification of the independent effect of 170 each individual stressor (i.e. HEAT-No MF; COLD-No MF; NEUTRAL-MF), as well as of 171 their interaction (i.e. HEAT-MF; COLD-MF), on both simple and complex manual tasks.

172 It should be noted that magnitude of heat stress (i.e. $60 \mathrm{~min}$ of passive exposure in moderate 173 heat \& humidity) was known to likely be insufficient to impair psychomotor function and 174 hence manual dexterity; yet the question of more relevance during the HEAT trials was 175 whether addition of mental fatigue would elicit impairments in dexterity.

\section{Thermal stress}

178 To induce hot and cold thermal stress, we passively exposed participants to a hot, a thermo179 neutral, and a cold climate, characterised by an ambient temperature of 37,21 and $7^{\circ} \mathrm{C}$, 180 respectively, with a constant relative humidity of $40 \%$. We chose these ambient temperatures 181 for several reasons. First and foremost, we wanted to ensure that the heat and cold exposures 182 selected for the 60-min trials would be sufficient to induce large changes in hand skin 183 temperature (e.g. reducing hand temperature to $\sim 20^{\circ} \mathrm{C}$ ), which was confirmed during pilot 
184 studies. Second, we wanted to replicate average ambient temperatures likely to be 185 experienced in an occupational setting relevant for the purpose of this study such as a military 186 operation in Afghanistan. We confirmed that the average temperature during the coldest part 187 of the year in Helmand province in Afghanistan is $\sim 7^{\circ} \mathrm{C}(\sim 40 \% \mathrm{RH})$, whereas the average 188 temperature during the hottest part of the year in the same province is $\sim 37^{\circ} \mathrm{C}(\sim 40 \% \mathrm{RH})$ 189 (World Weather Online.com). Mental fatigue

192 In order to generate prolonged periods of demanding cognitive activity (Desmond et al. 2001; 193 Job et al. 2001) that would be sufficient to induce a state of mental fatigue at the end of the mental fatigue trials, participants underwent a 35-min cognitive test battery, consisting of 5 separate tasks, each lasting $7 \mathrm{~min}$. The first three tasks were arithmetic calculations (based on the Key stage 2 of the UK national curriculum (17), followed by The Stroop Colour and Word Test (37), and the McKinsey problem solving test (34). All tests were written tests,

198 performed using a sheet of paper and a pencil provided to participants. To avoid a learning effect between trials, while maintaining the same level of cognitive demands, each participant performed three different versions of the same five tasks (e.g. different papers from Key stage 2) during each of the three mental fatigue trials they took part in. The tests were performed under time pressure, and verbal encouragement was given to complete them within the time allocated. The combination of time pressure and cognitive demand provided by these tests was deemed appropriate to induce a state of mental fatigue, which was later confirmed by both objective (i.e. reaction time) and subjective measures (i.e. self-reports of mental fatigue via a Likert scale). During the no-mental fatigue trials, participants watched non-stimulating videos.

\section{Experimental protocol}

210 Participants arrived at the laboratory on testing days, they changed into shorts and short 211 sleeve t-shirt and underwent preliminary measurements and preparation. They then 212 underwent a full familiarization with testing procedures, including the manipulative tasks (i.e. 213 several mock trials were allowed until participants confirmed confidence in the task), and the 214 assessments of mental fatigue (i.e. reaction time and Likert scale).

215 Following the familiarisation, participants self-inserted a rectal probe $13 \mathrm{~cm}$ passed the anal 216 sphincter (BlueTemp temperature probe, $\mathrm{UK}$ ) to record $\mathrm{T}_{\mathrm{rec}}$ as an index of core temperature. 217 A skin thermistor (Grant, UK) was taped to the centre of the hairy portion of the dominant 
218 hand with hypoallergenic medical tape $(3 \mathrm{M}, \mathrm{UK})$ to record local $\mathrm{T}_{\text {hand. Similarly, six }}$ 219 additional skin thermistors were taped to the centre of the forehead, the chest, the abdomen, 220 the scapula, the tricep and forearm, to record local skin temperatures for the calculation of a 221 simple mean of upper body skin temperature ( $\left.\mathrm{T}_{\text {upper body }}\right) . \mathrm{T}_{\text {rec }}, \mathrm{T}_{\text {hand }}$, and $\mathrm{T}_{\text {upper body }}$ were 222 recorded at $2 \mathrm{~Hz}$ via a dedicated data acquisition system (USB- Temp, MCCdaq, USA) and 223 custom-written software (DASYLab, MCCdaq, USA), and then averaged every 5 min for the 224 duration of the 60-min trials. At this point, participants moved into the climatic chamber, 225 which had been previously regulated to the air temperature and humidity required for the 226 specific 60-min trial. Participants took $5 \mathrm{~min}$ to enter the chamber and assume a seated 227 position on a small desk, where testing equipment was placed by accompanying the investigator. The following $55 \mathrm{~min}$ consisted of: a. baseline fatigue assessment and manipulation tasks (10 min); b. cognitive test battery or watching non-stimulating videos (35

$230 \mathrm{~min})$; c. post-test fatigue assessment and manipulation tasks (10 $\mathrm{min})$.

231 Once seated in the chamber, participants first performed 3 consecutive reaction time tests, 232 consisting of having to tap a box on a computer screen with their dominant index finger as 233 fast as they could when this turned from red to green (click test; Humanbenchmark.com, 234 2017). Reaction time was recorded in milliseconds and the average of 3 attempts was 235 calculated. Second, participants had to report on a 5-point Likert scale [1: Not mentally 236 fatigued; 2: Slight mental fatigue; 3: Mentally drained; 4: Mentally fatigued; 5: Mentally 237 exhausted; note: this was modified from the 12-point mental fatigue scale $(24 ; 18)]$ their 238 current mental fatigue state. Third, participants performed the pre-test manual tasks. The 239 O'Connor dexterity test consisted in having to place as many pins as possible in each hole of 240 the O'Connor board in 3 min (note: participants were instructed to place three pins per hole). 241 Upon completion of the O'Connor dexterity test, participants performed a modified version 242 of the Hand-Tool Dexterity test. This test consisted of having to unscrews and screw as many 243 horizontal hex nuts as possible in $3 \mathrm{~min}$. Nuts were screwed on 23 horizontal bolts mounted 244 on a wooden board. The number of pins and nuts were used as performance indicators for complex and simple manual tasks, respectively. At this point, and depending on the trial, participants underwent either the cognitive test battery or watched non-stimulating videos. Upon completion of this period, participants underwent again the objective and subjective assessment of mental fatigue, as well as the two manipulative tasks. Once those tasks were completed, participants exited the climatic chamber and were de-instrumented. 
252 To assess the independent and interactive effects of thermal stress and mental fatigue on 253 physiological and perceptual responses, and on manual performance, we first calculated the 254 difference (i.e. $\Delta$ ) between baseline and post-test assessments for each parameter and for each 255 individual participant. While units of measures were maintained for physiological and 256 perceptual parameters in baseline vs. post-trial differences (e.g. changes in $\mathrm{T}_{\text {hand }}$ were 257 expressed as $\Delta^{\circ} \mathrm{C}$ ), participants' manual performance was calculated as a percentage 258 difference between baseline and post-test in each trial.

259 First, we analysed differences in baseline (i.e. pre-test) $T_{\text {rec }}, T_{\text {hand }}, T_{\text {upper body }}$, reaction time, 260 subjective fatigue, fine and gross manipulative performance, by means of 1-way repeated 261 measures ANOVAs (i.e. 6 levels, one for each experimental trial). Second, we analysed $262 \Delta \mathrm{T}_{\text {rec }}, \Delta \mathrm{T}_{\text {hand }}, \Delta \mathrm{T}_{\text {upper body }}, \Delta$ reaction time, $\Delta$ subjective fatigue, $\Delta$ performance in fine and gross 263 manipulation (\%), for the independent and interactive effect of thermal stress (3 levels: 264 NEUTRAL vs. HEAT vs. COLD) and mental fatigue (2 levels: MF vs. No-MF), by means of 265 2-way repeated measure ANOVAs.

266 Post-hoc analyses were conducted with Tukey's multiple comparison tests. Regarding the 267 manual tasks, we compared participants' performance in each experimental trial including 268 either thermal stress, mental fatigue or both, with the performance in the NEUTRAL-No MF 269 (i.e. control) trial.

270 Data are reported as means, standard deviation (SD), and 95\% Confidence Intervals (CI). 271 Observed power was computed using alpha=0.05. Statistical analysis was performed using 272 GraphPad Prism (version 8.0; GraphPad Software, La Jolla, CA, USA).

\section{Results}

\section{Body temperatures}

276 Baseline (i.e. pre-test) $\mathrm{T}_{\text {rec }}, \mathrm{T}_{\text {hand }}, \mathrm{T}_{\text {upper body }}$ did not differ amongst the six trials (Tab.1).

277 First, we found that neither thermal stress $\left(\mathrm{F}_{(2,12)}=1.051 ; \mathrm{p}=0.380\right.$; explained variance $\left.4.5 \%\right)$, 278 nor mental fatigue $\left(\mathrm{F}_{(1,6)}=1.386 ; \mathrm{p}=0.284\right.$; explained variance $\left.4.1 \%\right)$ had a significant effect 279 on $\Delta \mathrm{T}_{\text {rec }}$ (Fig. 2A).

280 Second, we found that thermal stress $\left(\mathrm{F}_{(2,12)}=44.96 ; \mathrm{p}<0.0001\right.$; explained variance $\left.75.6 \%\right)$, 281 but not mental fatigue $\left(\mathrm{F}_{(1,6)}=1.344 ; \mathrm{p}=0.290\right.$; explained variance $\left.0.3 \%\right)$, had a significant 282 effect on $\Delta \mathrm{T}_{\text {hand. }}$. No interactions occurred between thermal stress and mental fatigue $\left(\mathrm{F}_{(2}\right.$, $\left.283{ }_{12}\right)=0.20 ; \mathrm{p}=0.820$; explained variance $0.2 \%$ ). When collapsed over mental fatigue trials and 284 compared to the NEUTRAL trial, $\Delta \mathrm{T}_{\text {hand }}$ was $-7.5^{\circ} \mathrm{C}([95 \% \mathrm{CI}:-10.7,-4.4] ; \mathrm{p}<0.0001)$ in the $285 \mathrm{COLD}$, while it was $+3.3^{\circ} \mathrm{C}([95 \% \mathrm{CI}:+0.2,+6.5]$; $\mathrm{p}<0.0001)$ in the HEAT (Fig. $\left.2 \mathrm{~B}\right)$. When 
286 expressed in absolute terms, post-test $\mathrm{T}_{\text {hand }}$ corresponded to $22.7 \pm 2.5^{\circ} \mathrm{C}$ during $\mathrm{COLD}$ trials, 287 and $34.7 \pm 1.1^{\circ} \mathrm{C}$ during HEAT trials.

288 Third, we found that thermal stress $\left(\mathrm{F}_{(2,12)}=116.6 ; \mathrm{p}<0.0001\right.$; explained variance $\left.85.1 \%\right)$, but not mental fatigue $\left(\mathrm{F}_{(1,6)}=0.281 ; \mathrm{p}=0.615\right.$; explained variance $\left.0.1 \%\right)$, had a significant effect

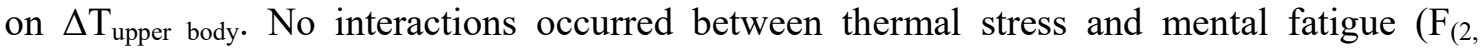
12) $=0.08 ; \mathrm{p}=0.917$; explained variance $0.1 \%$ ). When collapsed over mental fatigue trials and compared to the NEUTRAL trial, $\Delta \mathrm{T}_{\text {upper body }}$ was $-4.4^{\circ} \mathrm{C}([95 \% \mathrm{CI}:-5.6,-3.2] ; \mathrm{p}<0.0001)$ in the COLD, while it was $+2.2^{\circ} \mathrm{C}([95 \% \mathrm{CI}:+1.0,+3.4]$; $\mathrm{p}<0.0001)$ in the HEAT (Fig. $\left.2 \mathrm{C}\right)$. Table 2 reports post-trial data for $\mathrm{T}_{\text {rec }}, \mathrm{T}_{\text {hand, }}$, and $\mathrm{T}_{\text {upper body. }}$

All in all, these results indicated that our thermal stress conditions were effective in inducing large changes in local skin temperature of the hand and in upper body skin temperature, independently of the presence of mental fatigue.

\section{Objective and subjective measures of mental fatigue}

300 Baseline (i.e. pre-test) reaction time and subjective fatigue did not differ amongst the six 301 trials (Tab.1).

302 First, we found that mental fatigue $\left(\mathrm{F}_{(1,6)}=9.44 ; \mathrm{p}=0.021\right.$; explained variance $\left.21.4 \%\right)$, but not 303 thermal stress $\left(\mathrm{F}_{(2,12)}=0.45 ; \mathrm{p}=0.646\right.$; explained variance $\left.2.6 \%\right)$, had a significant effect on $304 \Delta$ reaction time (Fig. 3A). No interactions occurred between thermal stress and mental fatigue $305\left(\mathrm{~F}_{(2,12)}=1.38 ; \mathrm{p}=0.288\right.$; explained variance $\left.4.1 \%\right)$. When collapsed over thermal stress trials, 306 the difference in $\Delta$ reaction time between No-MF and MF trials was $+33 \mathrm{~ms}([95 \% \mathrm{CI}:+7$, $307+59] ; \mathrm{p}<0.0001$ ). Given that baseline reaction times were on average $352 \mathrm{~ms}$ (see Tab. 1), this 308 difference corresponded to $\sim 10 \%$ increase in reaction time because of the cognitive test 309 battery administered during MF trials.

310 Second, we found that mental fatigue $\left(\mathrm{F}_{(1,6)}=1405 ; \mathrm{p}<0.0001\right.$; explained variance $\left.86.9 \%\right)$, but 311 not thermal stress $\left(\mathrm{F}_{(2,12)}=0.84 ; \mathrm{p}=0.452\right.$; explained variance $\left.0.8 \%\right)$, had a significant effect on $312 \Delta$ subjective fatigue (Fig. 3B). No interactions occurred between thermal stress and mental 313 fatigue $\left(\mathrm{F}_{(2,12)}=1 ; \mathrm{p}=0.396\right.$; explained variance $\left.0.8 \%\right)$. When collapsed over thermal stress 314 trials, the difference in $\Delta$ subjective fatigue between No-MF and MF trials was +2.5 scale 315 points $([95 \% \mathrm{CI}:+2.3,+2.7]$; $\mathrm{p}<0.0001)$. Given that all participants reported a mental fatigue 316 of " 1 - Not Mentally fatigued" at the beginning of all trails (see Tab. 1), this difference 317 indicated that participant reported being between " 3 - Mentally drained" and " 4 - Mentally

318 fatigued" at the end of MF trials. Table 2 reports post-trial data for reaction time and 319 subjective fatigue. 
320 All in all, these results indicated that our mental fatigue conditions were effective in inducing

321 a state of mental fatigue, which was confirmed by both objective (i.e. rection time) and

322 subjective measures (i.e. self-reports), and which was independent of thermal stress.

323

\section{Manual performance}

325 Baseline performance in the complex and simple tasks did not differ amongst the six trials 326 (Tab.1).

327 First, we found that mental fatigue $\left(\mathrm{F}_{(1,6)}=54.4 ; \mathrm{p}=0.0003\right.$; explained variance $\left.48.7 \%\right)$, but not 328 thermal stress $\left(\mathrm{F}_{(2,12)}=2.88 ; \mathrm{p}=0.094\right.$; explained variance $\left.6.6 \%\right)$, had a significant effect on $329 \Delta$ complex manual performance. Also, we found a significant interaction between thermal 330 stress and mental fatigue $\left(\mathrm{F}_{(2,12)}=4.24 ; \mathrm{p}=0.040\right.$; explained variance $\left.8.9 \%\right)$. When compared 331 to NEUTRAL-No MF, manual performance in the complex task decreased by: $-22 \%$ $332([95 \% \mathrm{CI}-39,-5] ; \mathrm{p}=0.012)$ in the COLD-No MF; by $-16 \%([95 \% \mathrm{CI}-33,+1] ; \mathrm{p}=0.075)$ in the 333 HEAT-No MF; by $-36 \%([95 \%$ CI -53, -19]; p=0.0002) in the NEUTRAL-MF; by $-34 \%$ 334 ([95\%CI -52,-17]; $\mathrm{p}=0.0003)$ in the COLD-MF; and by $-36 \%([95 \% \mathrm{CI}-53,-19] ; \mathrm{p}=0.0002)$ 335 in the HEAT-MF (Fig. 4). These results indicated that mental fatigue alone induced greater 336 detriments on dexterity in the complex task (i.e. $36 \%$ performance drop) than cold stress 337 alone (i.e. $22 \%$ performance drop), and that when interacting with either cold or heat stress, 338 mental fatigue decreased manual performance by an extent equal (i.e. $\sim 35 \%$ combined 339 performance drop) to what observed when fatigue acted alone.

340 Second, we found that mental fatigue $\left(\mathrm{F}_{(1,6)}=28.9 ; \mathrm{p}=0.002\right.$; explained variance $\left.36.1 \%\right)$, but 341 not thermal stress $\left(\mathrm{F}_{(2,12)}=0.81 ; \mathrm{p}=0.468\right.$; explained variance $\left.3.9 \%\right)$, had a significant effect on

$342 \Delta$ simple manual performance. No interactions occurred between thermal stress and mental 343 fatigue $\left(\mathrm{F}_{(2,12)}=0.78 ; \mathrm{p}=0.476\right.$; explained variance $\left.2.2 \%\right)$. When collapsed over thermal stress 344 trials, the difference in $\Delta$ simple manual performance between No-MF and MF trials was $34530 \%$ ([95\%CI: $-43,-16] ; \mathrm{p}=0.002)$. Table 2 reports post-trial data for the complex and simple 346 tasks.

347 All in all, these results indicated that mental fatigue was the main inducer of the observed 348 detriments in dexterity during the simple manual task (i.e. 30\% performance drop), and that 349 these detriments were independent of thermal stress.

\section{Discussion}

352 The aim of this study was to determine the independent and interactive effects of thermal 353 stress and mental fatigue on manual dexterity. In assessing changes in manual performance 
354 during complex and simple tasks following on single and combined exposures to heat, cold, 355 and mental fatigue, we hypothesized that thermal stress and mental fatigue would interact 356 synergistically in reducing manual dexterity in healthy young adults.

357 With regards to the complex manual task, and contrary to our hypothesis, we found that, 358 while thermal stress and mental fatigue interacted in reducing manual dexterity (i.e. $\sim 35 \%$ 359 performance decrement), their mode of interaction was not synergistic. In fact, stressors 360 interaction followed a pattern whereby mental fatigue increased the individual impact of cold 361 (i.e. $22 \%$ performance decrement) and heat (i.e. $15 \%$ performance decrement), yet by an 362 extent no greater than that induced by mental fatigue alone (i.e. $36 \%$ performance 363 decrement). With regards to the simple manual task, we found that mental fatigue was the 364 main inducer of the observed detriments in dexterity (i.e. 30\% performance drop), with these 365 detriments being independent of thermal stress.

\section{Independent effects of thermal stress}

368 When considering the effects of thermal stress, our findings are in line with previous single369 stressor studies. Our observed $22 \%$ performance decrement in dexterity during the complex 370 task resulting from hand skin temperature dropping to an absolute value $\sim 23^{\circ} \mathrm{C}\left(\Delta-7.5^{\circ} \mathrm{C}\right)$, is

371 in line with previous results that have demonstrated an increase in hand numbness and a 372 decrease in finger dexterity at finger skin temperature between 22 and $14^{\circ} \mathrm{C}(9 ; 38)$. It is 373 important to note that we measured hand skin temperature at the centre of the back of the 374 hand. Given the large capacity for fast vaso-constriction (and -dilation) of the glabrous 375 portion of the fingers (39), one would expect that our recorded drops (and rises) in hand skin 376 temperature provide an underestimation of the actual extent of finger skin cooling (and 377 heating) occurring during our experiments. It is well-known that whole-body exposures to 378 cold increase proximal-to-distal differences in skin temperature, and that drops in local skin 379 temperature are more pronounced as one moves from to central body parts to fingers and toes 380 (e.g. as confirmed in our study when comparing $\Delta \mathrm{T}_{\text {hand }}$ vs. $\Delta \mathrm{T}_{\text {sk-upper body }}$ (39). Hence, it 381 appears likely that fingers' skin temperature may have dropped well below our recorded hand 382 temperature of $\sim 23^{\circ} \mathrm{C}$ and within the 22 to $14^{\circ} \mathrm{C}$ range previously shown to be associated 383 with decreased hand dexterity $(9 ; 38)$. Mechanistically, changes in manual performance due 384 to hand and fingers cooling arise from loss of cutaneous sensitivity (35), secondary to 385 conduction slowing in cold cutaneous nerve afferents $(10 ; 41)$. Given that conduction 386 velocity in cutaneous nerve fibers decreases linearly when skin temperature drops from 36 to $38723^{\circ} \mathrm{C}(10)$, it could be hypothesised that our observed decrements in fine manipulation were 
388 likely due to loss of cutaneous sensitivity. However, we did not measure peripheral 389 conduction velocities in the current study, and so this hypothesis remains speculative. Beside 390 cold-induced conduction slowing, cold-induced changes in joint mobility may have also

391 played a role in decreasing manual dexterity during the complex task in the current study.

392 Cold causes joints' synovial fluid to become viscous, which in turn reduces movements speed

393 and efficiency by increasing hand stiffness $(6 ; 21 ; 22)$, all of which could have contributed to

394 our observed cold-induced decrements in dexterity.

395 Finally, we did not observe any clear effect of thermal stress on dexterity during the simpler 396 manual task. This is not entirely surprising when considering that the cutaneous sensory 397 feedback that was likely impaired by thermal (and particularly cold) stress in the current 398 study is generally more important for finer and more complex sensorimotor actions, such as 399 those required when participants had to pinch-grip, move and accurately place each pin in 400 their respective small hole in the O'Connor test board (15b, 21, 36b). In fact, increases in 401 local tissue temperature can improve simpler, gross motor function (particularly during whole 402 body exercise) and these effects underlies the classic benefits of pre-exercise warm up on 403 subsequent motor performance (15).

\section{Independent effects of mental fatigue}

406 Regarding mental fatigue, it appears clear from our findings that this stressor had a prominent role in reducing manual performance. Specifically, mental fatigue explained $\sim 49 \%$ and $\sim 36 \%$

408 of observed variance during complex and simple manual tasks whereas thermal stress 409 explained only $6 \%$ and $4 \%$ of the variance, respectively.

410 Albeit lasting only $35 \mathrm{~min}$, our cognitive test battery appeared effective in inducing both a 411 subjective state of mental fatigue, as well as an objective $\sim 10 \%$ reduction in reaction time 412 during a task requiring visuo-motor coordination of the hand analogous to what required by 413 the manipulative tasks we administered (see Fig.3). The increase in both objective and 414 subjective measures of mental fatigue indicated that our cognitive test battery elicited a 415 significant level of cognitive load and perceived strain over the course of the MF trials.

416 Current theories on the effects of mental fatigue on physical performance suggest that this 417 psychobiological state may limit exercise tolerance via higher perceived exertion (32). 418 Furthermore, and in the context of short duration tasks presenting a certain level of cognitive 419 demand such as manual dexterity tasks, mental fatigue may hinder hand function $(3 ; 13)$ due 420 to its detrimental impact on cognitive and perceptual skills (14), including reaction time (26). 421 Mental fatigue has been previously demonstrated to negatively influence attention, action 
422 monitoring and cognitive control (2). Accordingly, we believe that our observed effects of 423 mental fatigue on manual performance are likely due to the induction of a significant 424 cognitive load, which may have been detrimental to neuropsychological parameters such as 425 attention, action monitoring, and reaction time, all of which are important for efficient 426 manual handling.

427 Although we did not record rates of perceived exertion, it could also be speculated that, 428 similarly to what is reported for submaximal whole-body exercise such as cycling (32), a 429 state of mental fatigue could have increased the perceived effort required by our manual 430 tasks, in turn leading to greater motor fatigue and worse overall performance given the same 431 task. It is important to note that mental fatigue seems to be similarly detrimental to both local 432 and whole-body exercise (40). For example, Bray et al. showed that cognitive effort induced 433 by a short-duration protocol negatively affected voluntary submaximal and maximal 434 contractions during a handgrip test $(3 ; 4)$. These authors reported greater proportional EMG 435 amplitude scores in the hand flexor muscles during the handgrip tasks in the participants who 436 performed the cognitive tasks. Bray et al.'s observations indicated that cognitive strain 437 contributed to peripheral fatigue (e.g. accumulation of metabolites) by inhibiting descending 438 neural activation of muscle motor units required to support the submaximal contraction $(3 ; 4)$.

439 Given that grip strength plays an important role in efficient manual handling, it cannot be 440 excluded that similar mental fatigue-induced changes in descending neural input to hand and 441 fingers muscles may have contributed to our observed decrements in dexterity under mental 442 fatigue states.

443

Combined effects of thermal stress and mental fatigue

445 Thermal stress and mental fatigue combine in many occupational and sporting settings (6), 446 and the potential for additive or synergistic interactions between these stressors may result in 447 magnifying hazards to individuals exposed to real world, multi stressors scenarios (27).

448 Within the constraints of our experiment (i.e. 60-min thermal stress exposure and 35-min 449 cognitive load), our findings indicated that, even when combined with thermal stress, mental 450 fatigue caused the greatest challenge to manual tasks. Importantly, and contrary to our initial 451 hypothesis, we found that, while thermal stress and mental fatigue interacted in reducing 452 dexterity during the complex task (i.e. $\sim 35 \%$ performance decrement), their mode of 453 interaction was not synergistic. In fact, stressors interaction followed a pattern whereby 454 mental fatigue increased the individual impact of cold (i.e. $\sim 22 \%$ performance decrement) 455 and heat (i.e. $\sim 15 \%$ performance decrement), yet by an extent no greater than that induced by 
456 mental fatigue alone (i.e. $\sim 36 \%$ performance decrement). This observation indicated a mode 457 of interaction between thermal stress and mental fatigue which followed the recently 458 proposed "most severe strain takes precedence" principle (27), whereby the more severe 459 strain "mental fatigue" takes precedence over the less severe strain "thermal stress". 460 According to this multi-stressor principle, the mode of interaction between stressors is 461 influenced by the impact magnitude of each individual stressor (e.g. thermal stress and 462 mental fatigue) (29). That is, the greater the effect of one stressor (e.g. mental fatigue), the 463 greater the probability that the detrimental effects of this stressor will take precedence over 464 the effects of the second stressor (e.g. thermal stress). This model fits well with our 465 observation that the interactive effects of thermal stress and mental fatigue were neither 466 additive nor synergistic, but that in fact their interactive-impact magnitude was equivalent to 467 the individual impact of the "most severe stressor" mental fatigue.

468 A state of mental fatigue in our participants is likely to have contributed to a less efficient 469 information's processing, secondary to a reduced allocation of attentional resources to task470 relevant cognitive processes (26). Mental fatigue may affect all stages of information 471 processing that receive modulatory top-down input, from stimulus processing to response 472 execution, and this notion is supported by studies that examined the effects of mental fatigue 473 on preparatory processes in different cognitive tasks $(1 ; 31)$. It could be therefore argued that 474 in our study, mental fatigue may have negatively affected the efficiency of stimulus 475 information processing relevant for initiating hand motor responses (26). The (likely) 476 "central effect" of mental fatigue appeared to be more severe than the (likely) "peripheral 477 effects" of thermal (cold) stress on hand sensorimotor function, and this differential impact 478 could explain why mental fatigue took precedence over thermal stress in limiting the 479 manipulative performance of our participants.

480 Mental fatigue has been recently reported to interact synergistically with heat stress during 481 cycling performance in the heat (36). Hence our findings may at first appear to contrast with 482 those of Otani et al. (36). However, it should be noted that in the study of Otani et al. (36) 483 both mental fatigue and heat stress induced independent severe strains, particularly as the 484 heat stress exposure resulted in a significant rise in core temperature prior to the cycling tests 485 in the heat. The severe mental and heat strain induced by the protocol of Otani et al. (36) 486 could have therefore produced the hyper-additive effects they observed. Our trials in the heat 487 did not induce any changes in core temperature, but in fact it only raised skin temperature. 488 Furthermore, it is likely that we did not observe a synergistic effect between heat and mental 489 fatigue because of the different performance task we adopted, and the differential effects that 
heat has on local vs. whole-body motor tasks and exercise. In contrast with the effects of a high core temperature on endurance whole body performance, local increases in tissue and muscle temperatures can indeed be beneficial to perform short-duration dynamic work such as the dexterity tasks we administered (21). Accordingly, we believe that our findings and those of Otani et al. (36) can be interpreted as two examples of how variations in the impact magnitude of a stressor may modulate its role in a multi-stressor interaction (27).

\section{Limitations}

498 We recognise some limitations to this study. First, our sample size is rather modest, although it appeared sufficient to demonstrate the effects of our chosen stressors, likely due to their large effect sizes (i.e. stressors induced performance decrements in the range of 20 to $30 \%$ ). Second, we did not implement performance tests that would clearly delineate central from peripheral effects of our stressors (i.e. simple- vs. choice-reaction time tasks using the same peripheral motor demands). Third, our experimental model did not involve a scenario where core temperature is shifted along with skin temperature. While evidence indicates that local changes in hand and finger temperatures play a greater role in worsening manual performance than changes in core temperature, we appreciate that increases in core temperature due to a combination of activity-induced metabolic heat and exposure to hot environments may have implications for manual dexterity and may be relevant to some occupational settings. Building on our findings, future studies should therefore consider mechanistic approaches to isolate peripheral from central effects in the interaction of thermal stress and mental fatigue, and how those may be modulated by changes in core temperature.

\section{Perspectives and significance}

514 From a fundamental standpoint, our study provides further support to the fact that human

515 integrative responses to multi-stressor scenarios follow a "worst-stressor-takes-precedence" 516 principle. From an applied point of view, our study supports the development of strategies 517 that primarily target the mitigation of mental fatigue to sustain manual performance in those 518 real world occupational (e.g. military, healthcare) and sport scenarios, which sees workers 519 and athletes being exposed to a combination of mental fatigue (e.g. induced by cognitive 520 stress or long shift work) and thermal stress (e.g. induced by exposure to extreme climates of 521 by wearing personal protective equipment).

\section{Conclusions}


524 We conclude that, within the constraints of our experiment (i.e. a combination of 60-min

525 passive exposures to air temperatures of 7 or $37^{\circ} \mathrm{C}$ with 35 -min of cognitive load), mental

526 fatigue posed the greatest challenge to manual dexterity in healthy young adults, even when

527 combined with heat and cold stress. Our findings highlight the important role that mental

528 fatigue can play in decreasing physical performance, both in isolation as well as when

529 interacting with other environmental stressors known to deteriorate manual dexterity such as

530 cold stress.

531

\section{Acknowledgements}

533 The authors thank Prof. George Havenith for the use of the climatic chambers at the

534 Environmental Ergonomics Research Centre (Loughborough University, UK).

535

536 References

537 1. Boksem MA, Meijman TF, Lorist MM. Mental fatigue, motivation and action 538 monitoring. Biological Psychology 72: 123-132, 2006.

539 2. Boksem MA, Tops M. Mental fatigue: Costs and benefits. Brain Research Review 59: $540 \quad 125-139,2008$.

541 3. Bray SR, Graham JD, Martin Ginis KA, Hicks AL. Cognitive task performance causes 542 impaired maximum force production in human hand flexor muscles. Biol Psychol 89(1): $543 \quad 195-200,2012$.

544 4. Bray SR, Martin Ginis KA, Hicks AL, Woodgate J. Effects of self-regulatory strength 545 depletion on muscular performance and EMG activation. Psychophysiology 45: 337-343, 5462008.

547 5. Broadbent D. Differences and interactions between stresses. Q J Exp Psychol 15: $205-$ $548 \quad 211,1963$.

549 6. Cheung SS, Lee JKW, Oksa J. Thermal stress, human performance, and physical 550 employment standards. Appl. Physiol. Nutr. Metab. 41: 148-164, 2016.

7. Cheung SS, Montie DL, White MD, Behm D. Changes in manual dexterity following short-term hand and forearm immersion in $10{ }^{\circ} \mathrm{C}$ water. Aviat. Space Environ. Med. 74(9): 990-993, 2003.

8. Daanen HAM, Heus R. The effect of body temperature on CIVD. Concept Report IZF, 1993.

9. Daanen HAM, Wammes LJA, Vrijkotte TGM. Windchill and dexterity. Report IZF A7, 1993. 
558 10. De Jong RH, Hershey WN, Wagman IH. Nerve conduction velocity during hypothermia in man. Anaesthesiology 27(6): 805-810, 1966.

11. Desmond PA, Hancock PA. Active and passive fatigue states. Stress, Workload and Fatigue 455-465, 2001.

12. Ducharme MB, Brajkovic D, Frim J. The effect of direct and indirect hand heating on finger blood flow and dexterity during cold exposure. $J$ Thermal Biol 24(5-6): 391-396,

13. Duncan MJ, Fowler N, George O, Joyce S, Hankey J. Mental fatigue negatively influences manual dexterity and anticipation timing but not repeated high-intensity exercise performance in trained adults. Res Sports Med 23(1): 1-13, 2015.

14. Faber L, Maurits NM, Lorist MM. Mental fatigue affects visual selective attention. PLos One 7(10): e48073, 2012.

15. Fradkin AJ, Zazryn TR, Smoliga JM. Effects of warming-up on physical performance: a systematic review with meta-analysis. Journal of Strength and Conditioning Research 24(1): 140-148, 2010.

15b. Nadia Gaoua, Sebastien Racinais, Justin Grantham \& Farid El Massioui (2011) Alterations in cognitive performance during passive hyperthermia are task dependent, International Journal of Hyperthermia, 27:1, 1-9, DOI: 10.3109/02656736.2010.516305

16. Giesbrecht GG, Bristow GK. Decrement in manual arm performance during whole body cooling. Aviat Space Environ Med 63: 1077-1081, 1992.

17. Gov.UK. Key stage 2 mathematics. Available at: https://www.gov.uk/government/publications/key-stage-2-tests-2019-mathematics-testmaterials

18. Grant S, Aitchison T, Henderson E, et al. A comparison of the reproducibility and the sensitivity to change of visual analogue scales, Borg scales, and Likert scales in normal subjects during submaximal exercise. Chest 116: 1208-1217, 1999.

19. Hagger MS, Wood C, Stiff C, Chatzisarantis NLD. Ego depletion and the strength model of self-control: A meta-analysis. Psychological Bulletin 136: 495-525, 2010.

20. Hellström B. Local effects of acclimatization to cold in man. Universitetsforlaget, Oslo. 1965.

21. Heus R, Daanen HA, Havenith G. Physiological criteria for functioning of hands in the cold: a review. Appl Ergon 26: 5-13, 1995.

22. Hunter J, Kerr EH, Whillans MG. The relation between joint stiffness upon exposure to cold and the characteristics of synovial fluid. Can J Med Science 30: 367-377, 1952. 
23. Job RFS, Dalziel J. Defining fatigue as a condition of the organism and distinguishing it from habituation, adaptation and boredom. Stress, Workload and Fatigue 466-475, 2001.

24. Joshi A, Kale S, Chandel S, Pal DK. Likert scale: explored and explained. British Journal of Applied Science \& Tecnology 7(4): 396-403, 2015.

25. Kauranen K, Vanharanta H. Effects of hot and cold packs on motor performance of normal hands. Physiotherapy 83(7): 340-344, 1997.

26. Langner R, Steinborn MB, Chatterjee A, Sturm W, Willmes K. Mental fatigue and temporal preparation in simple reaction time performance. Acta Psychologica 133: 64-72, 2010.

27. Lloyd A, Havenith G. Interactions in human performance: an individual and combined stressors approach. Temperature 3:1-4, 2016.

28. Lloyd A, Hodder S, Havenith G. The interactive effect of cooling and hypoxia on forearm fatigue development. Eur J Appl Physiol 115: 2007-2018, 2015.

29. Lloyd A, Raccuglia M, Hodder S, Havenith G. Interaction between environmental temperature and hypoxia on central and peripheral fatigue during high-intensity dynamic knee extension. J Appl Physiol 120: 567-579, 2016.

30. Lockhart JM, Kiess HO, Clegg TJ. Effect of rate and level of lowered finger surface temperature on manual performance. J Appl Physiol 40(1): 106-113, 1975.

31. Lorist MM. Impact of top-down control during mental fatigue. Brain Research 1232: 113-123, 2008.

32. Marcora SM, Staiano W, Manning V. Mental fatigue impairs physical performance in humans. J Appl Physiol 106(3): 857-64, 2009.

33. McCormick F, Kadzielski J, Landrigan CP, et al. Surgeon fatigue: a prospective analysis of the incidence, risk, and intervals of predicted fatigue-related impairment in residents. Arch Surg 147(5):430-435, 2012.

34. McKinsey\&Company. McKinsey Problem Solving Test. Available at: https://www.mckinsey.com/ /media/mckinsey/careers\%20redesign/interviewing/main/pr oblem $\% 20$ solving\%20test $\% 20$ pdfs/practice-test-a.ashx

35. Morton R, Provins KA. Figure numbness after acute local exposure to cold. $J$ Appl Physiol 15(1): 149-154, 1960.

36. Otani H, Kaya M, Tamaki A, Watson P. Separate and combined effects of exposure to heat stress and mental fatigue on endurance exercise capacity in the heat. Eur J Appl Physiol 117: 119-129, 2017.

36b. Jacob F. Piil, Jesper Lundbye-Jensen, Steven J. Trangmar \& Lars Nybo (2017) 
626 Performance in complex motor tasks deteriorates in hyperthermic humans, Temperature, 627 4:4, 420-428, DOI: 10.1080/23328940.2017.1368877

628 37. Scarpina F, Tagini S. The Stroop Color and Word Test. Front Psychol 8: 557, 2017.

629 38. Schieffer RE, Kok R, Lewis MI, Meese GB. Finger skin temperature and manual 630 dexterity; some inter-group differences. Appl Ergonomics 15(2): 135-141, 1984.

631 39. Taylor NA, Machado-Moreira CA, van den Heuvel AMJ, Caldwell JN. Hands and 632 feet: physiological insulators, radiators and evaporators. Eur J Appl Physiol 114(10): $633 \quad 2037-2060,2014$.

634 40. Van Cutsem J, Marcora SM, De Pauw K, Bailey S, Meeusen R, Roelands B. The 635 effects of mental fatigue on physical performance: a systematic review. Sports Medicine $636 \quad 47: 1569-1588,2017$.

637 41. Vanggaard L. Physiological reactions to wet cold. Aviat Space Environ Med 46(2): 33$638 \quad 36,1975$.

639

640

641 
644 Table 1. Baseline (i.e. pre-test) $T_{\text {rec }}, T_{\text {hand }}, T_{\text {sk-upper body }}$, reaction time and subjective fatigue, 645 and fine and gross manipulative performance (i.e. number of pins correctly placed and 646 number of bolts screwed and unscrewed in $3 \mathrm{~min}$ ), for the 7 participants, for of each of the 6 647 experimental trials. Data are reported as means and standard deviations. p-values for the 648 independent effect of trial (1-way ANOVA) are also reported.

Table 2. Post-test $T_{\text {rec }}, T_{\text {hand }}, T_{\text {sk-upper body }}$, reaction time and subjective fatigue, and fine and gross manipulative performance (i.e. number of pins correctly placed and number of bolts screwed and unscrewed in $3 \mathrm{~min}$ ), for the 7 participants, for of each of the 6 experimental trials. Data are reported as means and standard deviations.

Figure 1. Experimental design outlining the six separate trials undertaken by each participant. By comparing participants' change in manipulative performance between preand post-test in each trial with the pre-to-post change in performance in the NEUTRAL-No MF trial (i.e. namely the control condition), this design allowed for the quantification of the independent effect of each individual stressor (i.e. HEAT-No MF; COLD-No MF; NEUTRAL-MF), as well as of their interaction (i.e. HEAT-MF; COLD-MF), on both fine and gross manipulation.

662

Figure 2. Mean $(\mathrm{N}=7)$ difference and $95 \% \mathrm{CI}$ for the $\Delta \mathrm{T}_{\text {rec }}(\mathrm{A}), \Delta \mathrm{T}_{\text {hand }}(\mathrm{B})$, and $\Delta \mathrm{T}_{\text {upper body }}$

664 (C). Data were collapsed over mental fatigue trials and compared to the NEUTRAL trial to 665 visualise the independent effect of HEAT and COLD stress on body temperatures. * 666 corresponds to $\mathrm{p}<0.05$ for 2-way ANOVA post-hoc analyses.

668 Figure 3. Mean $(\mathrm{N}=7)$ difference and 95\% CI for the reaction time (A) and subjective fatigue 669 (B). Data were collapsed over thermal stress trials to visualise the independent effect of 670 mental fatigue on objective and subjective measures. * corresponds to $\mathrm{p}<0.05$ for 2 -way 671 ANOVA main effect.

673 Figure 4. Mean difference $(\mathrm{N}=7)$ and $95 \%$ in manual performance for the complex task. Data 674 for the 5 experimental conditions (i.e. COLD-MF, HEAT-MF, NEUTRAL-MF, COLD-No 675 MF, HEAT-No MF) were compared to the NEUTRAL-No MF trial (i.e. namely the control 
676 condition) to visualise the independent and interactive effects of HEAT and COLD, and of 677 MENTAL FATIGUE, on manual performance. * corresponds to $\mathrm{p}<0.05$ for 2 -way ANOVA 678 post-hoc analyses. 


\section{NEUTRAL-No MF NEUTRAL-MF COLD-No MF COLD-MF HEAT-No MF HEAT-MF}

$\begin{array}{rrrrrrrrrrrrrrr}\mathrm{T}_{\text {rec }}\left({ }^{\circ} \mathrm{C}\right) & 37.32 & \pm 0.21 & 37.11 & \pm 0.17 & 37.24 & \pm 0.18 & 37.29 & \pm 0.32 & 37.19 & \pm 0.27 & 37.26 & \pm 0.26 & (\mathrm{p}=0.560) \\ & & & & & & & & & & & & & & \\ \mathrm{T}_{\text {hand }}\left({ }^{\circ} \mathrm{C}\right) & 31.34 & \pm 2.65 & 30.15 & \pm 1.72 & 29.67 & \pm 2.87 & 29.45 & \pm 2.07 & 30.52 & \pm 2.81 & 30.29 & \pm 3.01 & (\mathrm{p}=0.584)\end{array}$




\begin{tabular}{|c|c|c|c|c|c|c|c|c|c|c|c|c|c|}
\hline $\mathrm{T}_{\text {upper body }}\left({ }^{\circ} \mathrm{C}\right)$ & 31.81 & \pm 0.80 & 31.26 & \pm 0.81 & 31.62 & \pm 0.99 & 31.53 & \pm 0.27 & 31.75 & \pm 1.25 & 32.02 & \pm 1.46 & $(p=0.580)$ \\
\hline Reaction time (ms) & 352 & \pm 38 & 357 & \pm 40 & 347 & \pm 32 & 348 & \pm 52 & 353 & \pm 52 & 357 & \pm 27 & $(p=0.886)$ \\
\hline $\begin{array}{r}\text { Subjective fatigue } \\
(1-5)\end{array}$ & 1.0 & \pm 0.0 & 1.0 & \pm 0.0 & 1.0 & \pm 0.0 & 1.0 & \pm 0.0 & 1.0 & \pm 0.0 & 1.0 & \pm 0.0 & $(p=1.000)$ \\
\hline $\begin{array}{r}\text { Fine manual task } \\
(\mathrm{N} \text { pins })\end{array}$ & 43.3 & \pm 6.2 & 49.4 & \pm 4.6 & 48.4 & \pm 8.8 & 48.9 & \pm 5.1 & 43.4 & \pm 6.7 & 49.3 & \pm 7.7 & $(p=0.200)$ \\
\hline $\begin{array}{r}\text { Gross manual task } \\
\text { (N bolts) }\end{array}$ & 42.3 & \pm 9.8 & 49 & \pm 9.6 & 42.3 & \pm 10.4 & 49.7 & \pm 4.3 & 45.7 & \pm 8.0 & 51 & \pm 4.0 & $(p=0.199)$ \\
\hline
\end{tabular}




\section{NEUTRAL-No MF NEUTRAL-MF COLD-No MF COLD-MF HEAT-No MF HEAT-MF}

$\begin{array}{rrrrrrrrrrrrr}\mathrm{T}_{\text {rec }}\left({ }^{\circ} \mathrm{C}\right) & 37.12 & \pm 0.22 & 36.96 & \pm 0.16 & 37.15 & \pm 0.22 & 37.25 & \pm 0.36 & 37 & \pm 0.18 & 37.23 & \pm 0.26 \\ & & & & & & & & & & & & \\ \mathrm{~T}_{\text {hand }}\left({ }^{\circ} \mathrm{C}\right) & 32.08 & \pm 1.72 & 30.78 & \pm 0.99 & 23.48 & \pm 3.19 & 21.93 & \pm 1.50 & 34.76 & \pm 1.42 & 34.65 & \pm 0.92\end{array}$




\begin{tabular}{|c|c|c|c|c|c|c|c|c|c|c|c|c|}
\hline $\mathrm{T}_{\text {upper body }}\left({ }^{\circ} \mathrm{C}\right)$ & 32.29 & \pm 0.75 & 31.7 & \pm 0.60 & 27.71 & \pm 2.49 & 27.52 & \pm 1.48 & 34.65 & \pm 0.43 & 34.46 & \pm 0.81 \\
\hline Reaction time (ms) & 359 & \pm 37 & 377 & \pm 34 & 350 & \pm 38 & 400 & \pm 48 & 360 & \pm 66 & 400 & \pm 42 \\
\hline $\begin{array}{r}\text { Subjective fatigue } \\
(1-5)\end{array}$ & 1.1 & \pm 0.4 & 3.6 & \pm 0.8 & 1.0 & \pm 0.0 & 3.8 & \pm 0.7 & 1.0 & \pm 0.0 & 3.4 & \pm 0.5 \\
\hline $\begin{array}{r}\text { Fine manual task } \\
(\mathrm{N} \text { pins })\end{array}$ & 48.1 & \pm 4.4 & 37.6 & \pm 2.6 & 44.1 & \pm 10.1 & 38.1 & \pm 7.5 & 41.9 & \pm 5.1 & 37.6 & \pm 5.1 \\
\hline $\begin{array}{r}\text { Gross manual task } \\
(\mathrm{N} \text { bolts })\end{array}$ & 46.7 & \pm 5.6 & 39.6 & \pm 4.0 & 41.1 & \pm 7.8 & 40.1 & \pm 8.6 & 53.3 & \pm 6.3 & 42.6 & \pm 7.6 \\
\hline
\end{tabular}




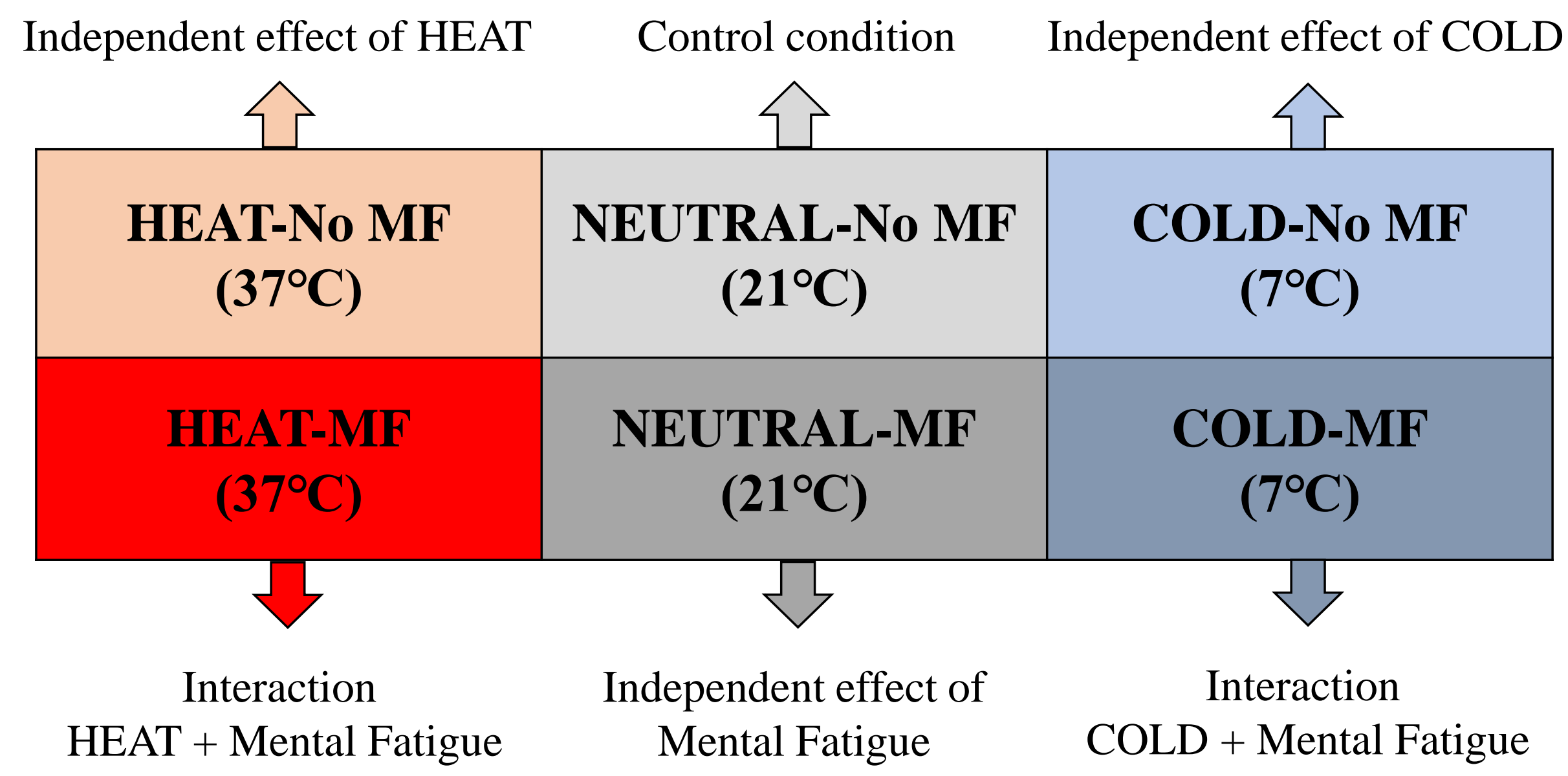



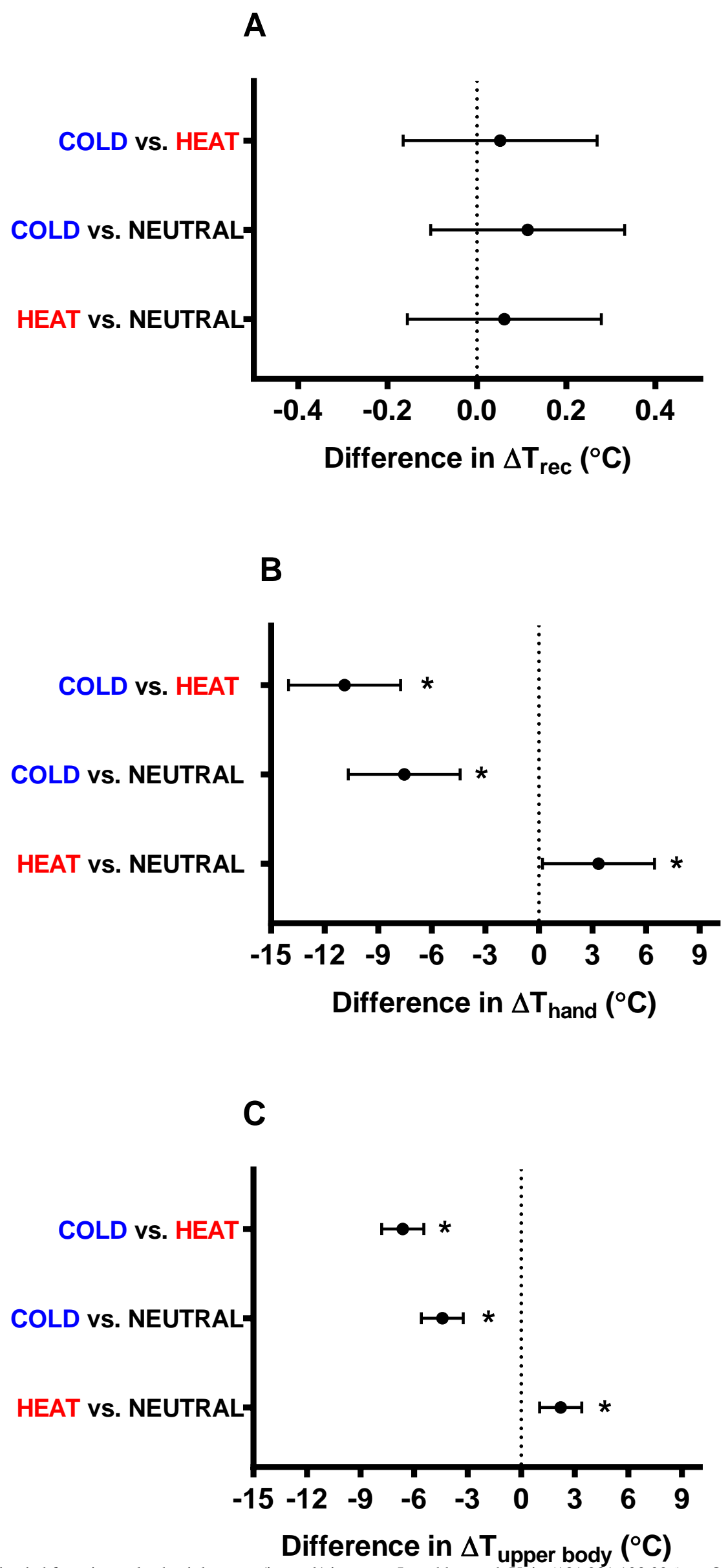

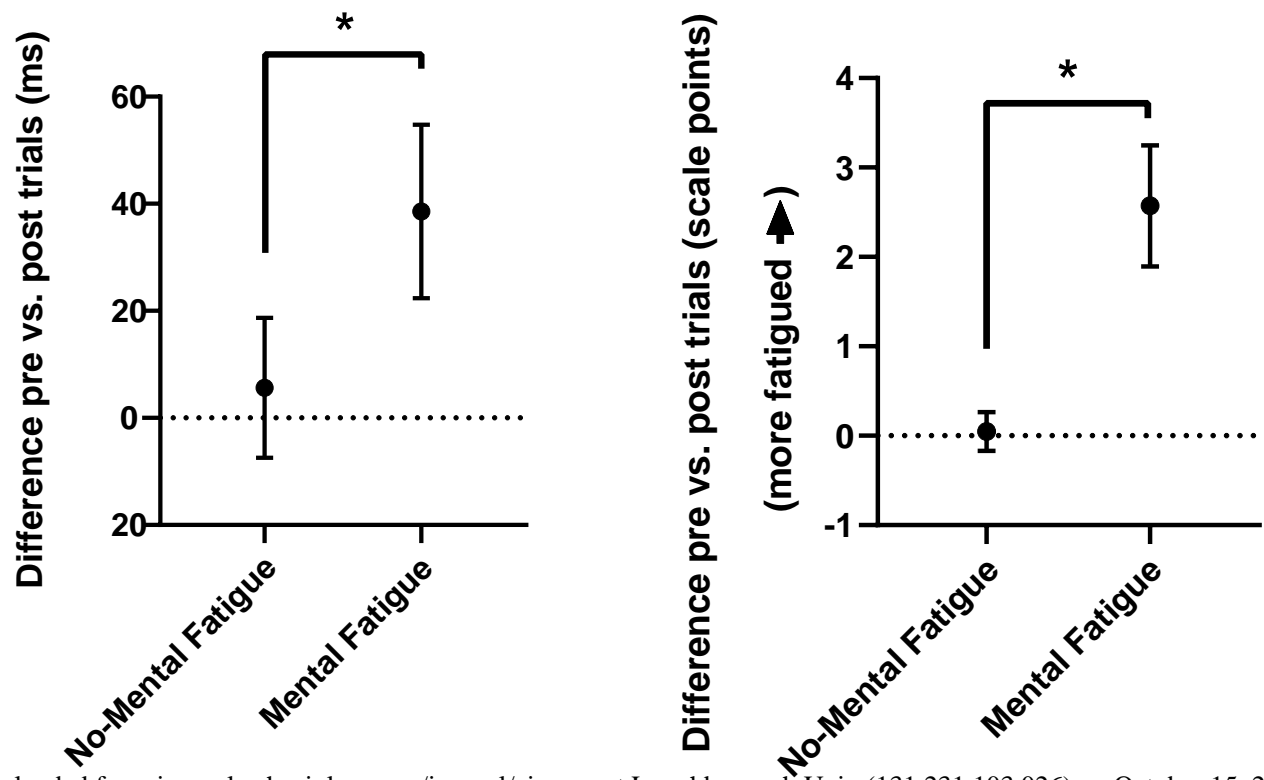

Downloaded from journals.physiology.org/journal/ajpregu at Loughborough Univ (131.231.103.026) on October 15, 2020. 
COLD-MF vs. NEUTRAL-No MF HEAT-MF vs. NEUTRAL-No MF NEUTRAL-MF vs. NEUTRAL-No MF COLD-No MF vs. NEUTRAL-No MF HEAT-No MF vs. NEUTRAL-No MF

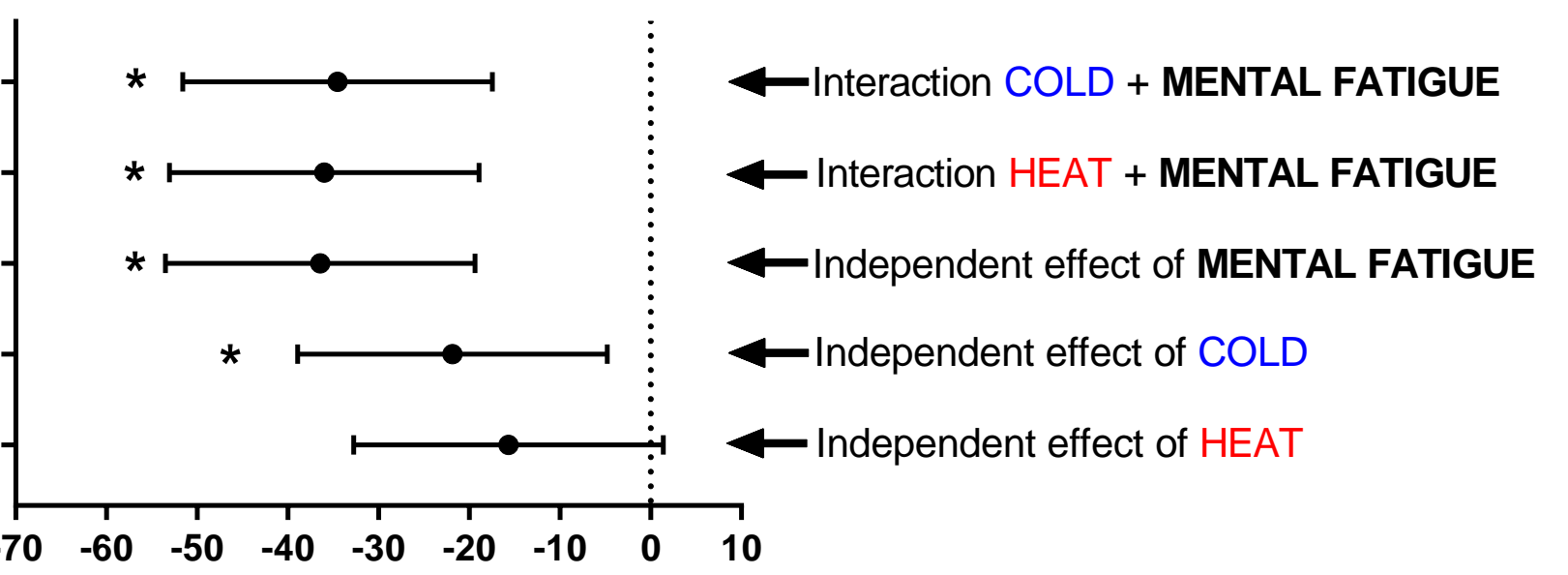

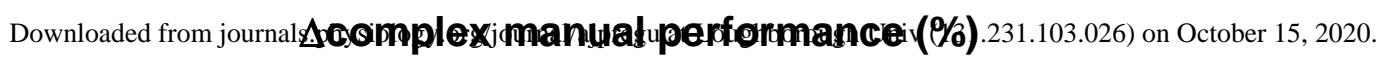

